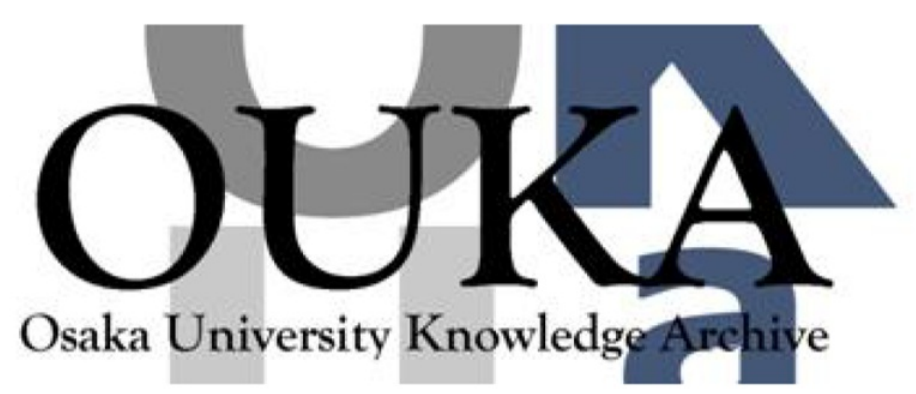

\begin{tabular}{|c|l|}
\hline Title & $\begin{array}{l}\text { Resonance ultrasound spectroscopy for measuring } \\
\text { elastic constants of thin films }\end{array}$ \\
\hline Author(s) & $\begin{array}{l}\text { Nakamura, Nobutomo; Ogi, Hirotsugu; Hirao, } \\
\text { Masahiko }\end{array}$ \\
\hline Citation & $\begin{array}{l}\text { Japanese Journal of Applied Physics. } 43 \text { p. 3115- } \\
\text { p. 3118 }\end{array}$ \\
\hline Issue Date & $2004-05-28$ \\
\hline oaire:version AM \\
\hline URL & https://hdl.handle. net/11094/84157 \\
\hline rights & \\
\hline Note & \\
\hline
\end{tabular}

Osaka University Knowledge Archive : OUKA

https://ir. Library. osaka-u. ac. jp/

Osaka University 


\title{
Resonance Ultrasound Spectroscopy for Measuring Elastic Constants of Thin Films
}

\author{
Nobutomo NAKAMURA, Hirotsugu OGI and Masahiko HIRAO \\ Graduate School of Engineering Science, Osaka University, 1-3 Machikaneyama, Toyonaka, Osaka 560-8531, Japan
}

\begin{abstract}
We show an advanced technique for measuring elastic constants $C_{i j}$ of thin films deposited on substrates. Thin films often show anisotropy between the in-plane and out-of-plane directions because of their columnar structure, residual stress, texture, and incohesive bond. Then, thin films show macroscopically transverse isotropy and have five independent $C_{i j}$. All the film $C_{i j}$ affect free-vibration resonance frequencies of the film/substrate layered specimen. Therefore, measuring the resonance frequencies permits us to determine the thin-film $C_{i j}$ with the other known parameters. In order to yield reliable $C_{i j}$ of thin films, we have to measure the resonance frequencies with sufficient accuracy and identify vibration modes of the measured resonance frequencies. We overcome these problems by developing a tripod and using a laser-Doppler interferometer, respectively. We applied the present technique to a copper thin film. Measured $C_{i j}$ are smaller than those of bulk and show elastic anisotropy. We attribute these features to the incohesive bond regions.
\end{abstract}

KEYWORDS: elastic constants, laser measurement, micromechanics, resonance ultrasound spectroscopy, thin films

\section{Introduction}

Determination of a thin film's elastic constants $C_{i j}$ is an important issue for designing devices such as surface-acoustic-wave (SAW) devices and microelectromechanical systems (MEMS). In addition, it enables us to evaluate micro- or nano-scale defects in the film, because they highly affect the film $C_{i j}$ through elastic softening.

Thin films often show anisotropy between the in-plane and out-of-plane directions because of their columnar structure, residual stress, texture, and incohesive bonds. Thus, their elastic constants show transverse isotropy (or hexagonal symmetry) and have five independent elastic constants. They are denoted by $C_{11}, C_{33}, C_{13}, C_{44}$, and $C_{66}$ when the $x_{3}$ axis is along the out-of-plane direction and the $x_{1}$ and $x_{2}$ axes lie in the in-plane direction. Many previous studies assumed that thin films are elastically isotropic and reported only the in-plane Young's modulus $E_{1}$ using the static bending test ${ }^{1)}$ and flexural vibration of a reed composed of a film/substrate layered plate. $^{2)}$ These methods always involve ambiguity caused by the mechanical contacts used to grip the specimen and to make the acoustic transduction. The Brillouinscattering technique ${ }^{3)}$ can deduce all the five elastic constants, but failed to detect the elastic anisotropy because it is insensitive to non-Rayleigh-wave acoustic modes.

In this study, we propose an acoustic method for determining thin film $C_{i j}$, which is a combination of resonance ultrasound spectroscopy (RUS) ${ }^{4,5)}$ and laserDoppler interferometry. The RUS method deduces thin film $C_{i j}$ from free-vibration resonance frequencies of a film/substrate layered specimen. Mode identification for the measured resonance frequencies is indispensable for successful determination. However, few studies achieved correct mode identification. Here, we use laser-Doppler interferometry to measure the displacement distributions on the specimen surface and to identify the vibration modes.

We applied the present method to a copper thin film deposited on monocrystal silicon. Measured $C_{i j}$ are smaller than those of bulk copper and show elastic anisotropy $\left(C_{11}>C_{33}\right)$. As the cause, we consider

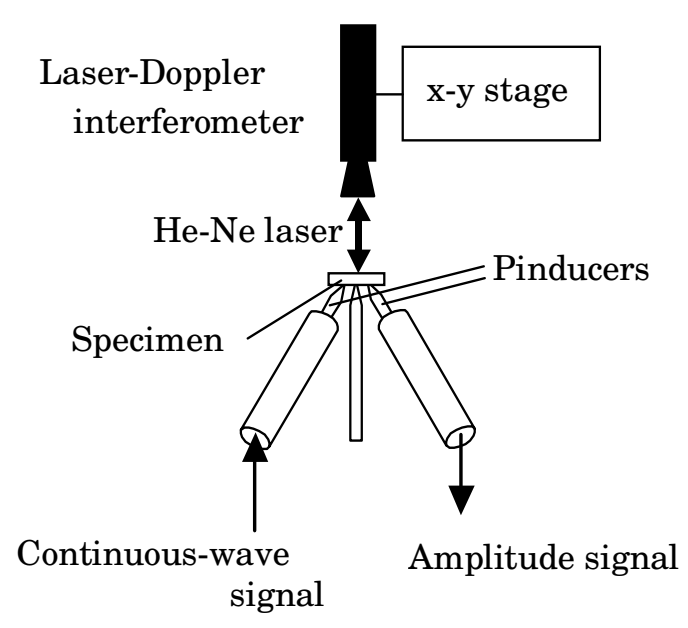

Fig. 1. Schematic of the RUS/laser measurement setup.

the effect of texture or incohesive bonds. From the Xray-diffraction measurement and micromechanics calculation, we concluded that the presence of the incohesive bonds is the principal cause.

\section{RUS/Laser Technique}

Free-vibration resonance frequencies of a rectangular parallelepiped specimen depend on dimensions, mass density, and all independent elastic constants. The elastic constants are inversely determined by measuring the free-vibration resonance frequencies, dimensions, and density. This method is called RUS. Similarly, for a film/substrate layered specimen, the film $C_{i j}$ is determined from the free-vibration resonance frequencies and dimensions, mass densities, and $C_{i j}$ of the substrate. However, because the film $C_{i j}$ contributes weakly to the resonance frequencies, normally as small as $1 \%$, we must measure the resonance frequencies with a high accuracy. Previous RUS studies ${ }^{4,5)}$ measured the resonance frequencies by sandwiching specimens between two transducers. This setup constrains the specimen's deformation and changes the resonance frequencies from those of ideal free vibration. In order to minimize this influence, 


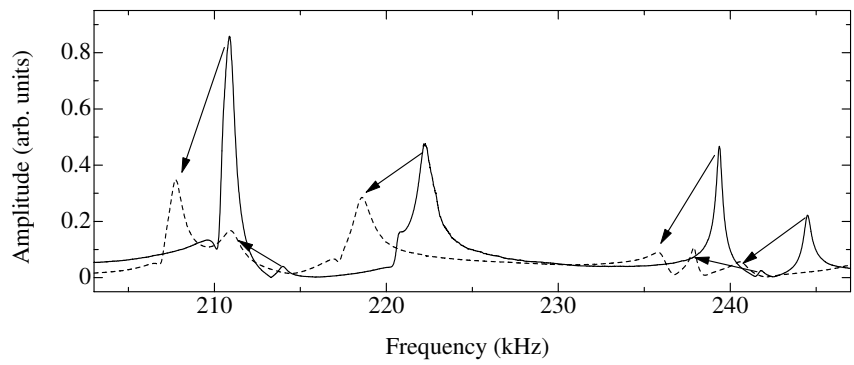

Fig. 2. RUS spectra of the monocrystal silicon substrate (12 ! $\left.10 ! \_0.2 \mathrm{~mm}^{3}\right)$ (solid line) and copper $(2.3 \mu \mathrm{m}) /$ silicon layered specimen (dashed line).

we developed a piezoelectric tripod, ${ }^{6-8)}$ which consists of two piezoelectric needle-type transducers and a support needle as shown in Fig. 1. One needle-type transducer generates a sinusoidal continuous-wave $(\mathrm{cw})$ vibration in the specimen and the other needle-type transducer detects the amplitude response. By sweeping the driving frequency and obtaining the amplitude as a function of the frequency, we obtain a resonance spectrum as shown in Fig. 2. The resonance frequencies are determined by fitting a Lorentzian function around the peaks. Contacts between the specimen and needle-type transducers are weak and stable because no coupling material is needed: only the specimen mass contributes to the acoustic coupling. Thus, the reproducibility of a resonance-frequency measurement is better than $10^{-4}$.

The inverse calculation requires calculation of the resonance frequencies of a solid specimen. For a non-layered rectangular parallelepiped specimen, we consider minimization of the Lagrangian:

$$
\delta \int_{V} L d V=0
$$

where

$$
L=\frac{1}{2}\left(C_{i j} S_{i} S_{j}-\rho \omega^{2} u_{i} u_{i}\right)
$$

denotes Lagrangian. The integration is taken over the volume $V$ of the solid. $\rho$ denotes the mass density, $\omega$ the angular frequency, $u_{i}$ the displacement in the $x_{i}$ axis, and $S_{i}$ the engineering strain. Because no analytical solution exists for the displacements in a rectangularparallelepiped solid subjected to a free vibration, the displacements are approximated by linear combinations of basis functions $\Psi_{k}$ :

$$
u_{i}\left(x_{1}, x_{2}, x_{3}, t\right)=\sum_{k} U_{k}^{i} \Psi_{k}^{i}\left(x_{1}, x_{2}, x_{3}\right) e^{j \omega t}
$$

Here, $U_{k}^{i}$ denote the expansion coefficients.

For a layered parallelepiped specimen, the formulation must include the discontinuous displacement gradients at the film/substrate interface, arising from their different moduli. Then, Heyliger ${ }^{9)}$ used different basis functions for the thickness direction $\left(x_{3}\right)$ and in-plane direction $\left(x_{1}\right.$ and $\left.x_{2}\right)$ as

$$
\Psi_{k}^{i}\left(x_{1}, x_{2}, x_{3}\right)=\eta_{m}^{i}\left(x_{3}\right) \zeta_{l}^{i}\left(x_{1}, x_{2}\right),
$$

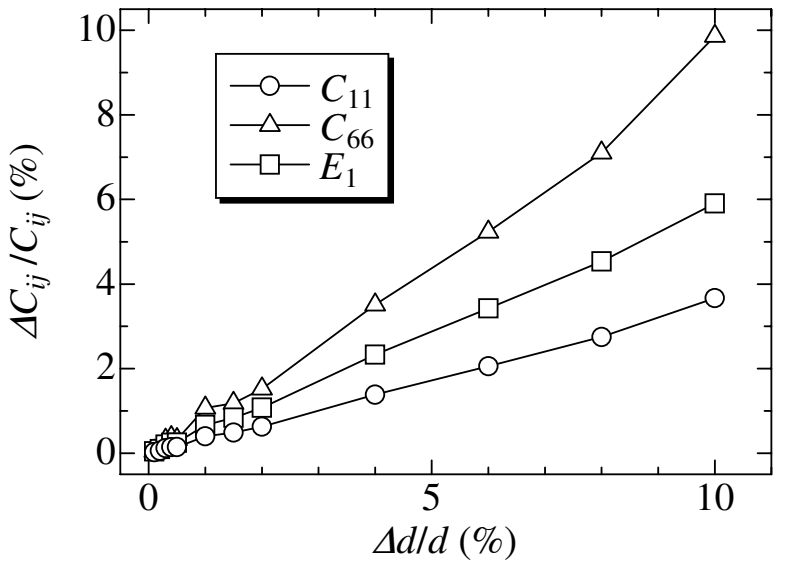

Fig. 3. Relationship between the film thickness error and errors in the resultant film $C_{i j}$.

where $\eta_{m}^{i}\left(x_{3}\right)$ denotes the one-dimensional Lagrangian interpolation polynomials and $\zeta_{l}^{i}\left(x_{1}, x_{2}\right)$ denotes power series $x_{1}^{p} x_{2}^{q}(p, q=0,1,2, ! \mathrm{D})$. Substituting eqs. (3) and (4) into eq. (1) results in the matrix form of

$$
\omega^{2}[\mathbf{M}]\{\mathbf{U}\}=[\mathbf{K}]\{\mathbf{U}\} .
$$

Here, $[\mathbf{M}]$ denotes the mass matrix associated with the kinetic energy of the system and $[\mathbf{K}]$ denotes the stiffness matrix associated with the potential energy of the system. $\{\mathbf{U}\}$ denotes the eigenvector composed of the expansion coefficients, which provide the displacement distributions in the specimen with eq. (3). Thus, the analysis is formulated in an eigenvalue problem and the resonance frequencies are obtained from the eigenvalues of the system. The thin film elastic constants are inversely determined by comparing the calculated resonance frequencies with the measurements. We used a standard least-squares-fitting procedure in Heyliger's method to determine a set of elastic constants that minimizes the differences between measured and calculated resonance frequencies. Such a calculation method was confirmed by Ogi et al.. ${ }^{10)}$

To determine the film $C_{i j}$ inversely from the resonance frequencies, correct correspondence of the observed and calculated resonance frequencies must be achieved. If the inverse calculation contains mode misidentification, the resulting $C_{i j}$ are physically meaningless. However, the mode identification has never been straightforward, because the resonance spectrum does not include any information on modes. We overcome this by mapping the out-of-plane displacements using a laser-Doppler interferometer (Fig. 1) and comparing them with the calculated displacement distributions.

\section{Measurement Accuracy}

There are two possible error sources in the determined film $C_{i j}$; (i) measurement error of the film thickness and, (ii) measurement error of the resonance frequencies. Here, we investigate their influences on the determined film $C_{i j}$. 
Table I. Measured and calculated elastic constants (GPa) of copper thin film deposited on a 0.2-mm monocrystal silicon substrate. $C_{66}=\left(C_{11}-C_{12}\right) / 2$.

\begin{tabular}{ccccc}
\hline & $\begin{array}{c}\text { Copper thin film } \\
(2.3 \mu \mathrm{m})\end{array}$ & $\begin{array}{c}\text { Hill averaging } \\
\text { (isotropic) }\end{array}$ & $\begin{array}{c}\text { Micromechanics } \\
\left.\text { (porosity }=6.2 \times 10^{-4}\right)\end{array}$ & $\begin{array}{c}(111) \\
\text { texture }\end{array}$ \\
\hline$C_{11}$ & $172.6 \pm 13.8$ & 197.7 & 169.7 & 213.3 \\
$C_{33}$ & $92.0 \pm 7.0$ & 197.7 & 94.8 & 237.9 \\
$C_{13}$ & $134.0 \pm 5.1$ & 103.0 & 49.4 & 87.1 \\
$C_{12}$ & $80.7 \pm 15.1$ & 103.0 & 75.0 & 111.7 \\
$C_{44}$ & - & 47.4 & 36.1 & 35.7 \\
$C_{66}$ & $45.9 \pm 6.1$ & 47.4 & 47.3 & 50.8 \\
\hline
\end{tabular}

\subsection{Measurement error of dimensions}

We determine the film thickness by its cross-sectional SEM observation. Because the measurement of the film thickness usually includes the largest error, we estimate its effect numerically by considering a copper/silicon layered model. The substrate is a monocrystal silicon substrate, measuring $12.000 ! \_10.000 ! \_0.200 \mathrm{~mm}^{3}$ with the (100) face normal to the out-of-plane direction. Copper film is $3 \mu \mathrm{m}$ thick. We calculated the free-vibration resonance frequencies of the layered specimen and determined the film $C_{i j}$ assuming various film-thickness errors $(d+\Delta d)$. Figure 3 shows the results. The errors in the resultant $C_{i j}$ are in proportion to the film thickness errors. The film-thickness variation in the cross-sectional observation is approximately $5 \%$ at most, indicating that errors caused by the film-thickness error in the $C_{i j}$ are less than $5 \%$.

\subsection{Measurement errors of resonance frequencies}

The measurement errors of the resonance frequencies significantly affect the resulting film $C_{i j}$. We estimate this $\left(\Delta C_{i j}\right)$ from the contributions of the $C_{i j}$ to the resonance frequencies $\left(\partial f / \partial C_{i j}\right)$ and measurement errors of the resonance frequencies $(\Delta f)$ as

$$
\Delta C_{i j}=\frac{1}{\partial f / \partial C_{i j}} \Delta f
$$

$\partial f / \partial C_{i j}$ is obtainable from the inverse calculation. ${ }^{5)}$ Thus, elastic constants with smaller contribution cannot be determined accurately.

\section{Elastic Constants of Copper Thin Film}

We applied the present method to a polycrystalline copper film deposited on a monocrystal silicon substrate $\left(12.019\right.$ !_10.099!_0.211 $\left.\mathrm{mm}^{3}\right)$. First, we measured the resonance frequencies of the substrate alone to determine the substrate $C_{i j}$. They agreed with well known values ${ }^{11)}$ within a $1 \%$ difference. Then, we deposited the copper film by the magnetron-sputtering method and measured the resonance frequencies of the $\mathrm{Cu} / \mathrm{Si}$ specimen. The pressure in the sputtering chamber was kept less than $5 ! 1^{-5}$ Torr prior to the sputtering; it increased up to 0.05 Torr during the sputtering because of the addition of high-purity argon gas. The biasing voltage was $400 \mathrm{~V}$ and the current was $200 \mathrm{~mA}$. The deposition rate was $3 \AA / \mathrm{s}$. The film thickness was $2.3 \mu \mathrm{m}$ and the Xray diffraction spectrum indicates a (111) texture. We calculated the lattice parameter of the copper thin film from the X-ray diffraction spectrum, which agreed with the bulk's value with a difference of $0.1 \%$. Also, SEM

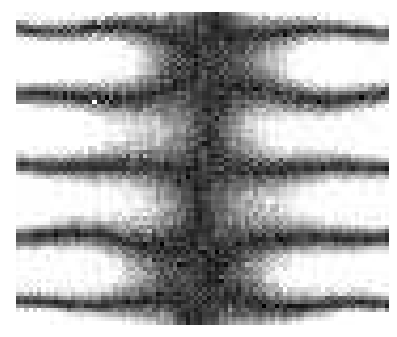

Measurement

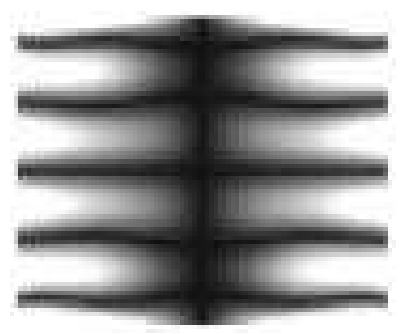

Calculation
Fig. 4. Measured (left) and calculated (right) displacement-amplitude distributions of $2.3-\mu \mathrm{m}$-thick copper/0.2-mm silicon vibrating at $151.4 \mathrm{kHz}$.

observation did not revealed any volume defects. Therefore, we used the mass density of bulk copper for the film. After the deposition, the resonance peaks shifted to lower frequencies as shown in Fig. 2. Figure 4 shows an example of the comparison between the measured and computed displacement distributions for the $\mathrm{Cu} / \mathrm{Si}$ specimen. We see excellent agreement between them, which ensures correct mode identification. Thus, we identified more than twenty resonance modes and entered them into the inverse calculation to determine the film $C_{i j}$.

Table I shows the determined copper thin film $C_{i j}$ along with the isotopic $C_{i j}$ calculated by Hill approximation using monocrystal data. We failed to determine the film $C_{44}$ because of its very small contributions to the resonance frequencies. Significant observations are (1) the $C_{33}$ is markedly smaller than $C_{11}$ and (2) the film $C_{i j}$ are smaller than those of the ideal isotropic $C_{i j}$. As a possible cause of this anisotropy, we consider (i) texture and (ii) local incohesive bonds. The observed X-raydiffraction spectrum indicates the (111) planes oriented preferentially parallel to the film surface. We calculated the macroscopic $C_{i j}$ of such a textured microstructure in which all the (111) planes of grains are aligned parallel to the $x_{3}$ surface with a random rotation around the $x_{3}$ axis. The Hill approximation was used for this calculation. The calculated $C_{i j}$ values appear in Table I. The $C_{33}$ of the copper film is larger than $C_{11}$ in the case of the (111) texture; this result is opposite to the observed results. Thus, the texture cannot be a dominant factor of the observed anisotropy.

Second, we consider the presence of incohesive bonds at grain boundaries by micromechanics modeling. For 
copper thin films, Schwaiger et al. ${ }^{12)}$ reported that thinner films are more fatigue-resistant and contain fewer and smaller extrusions than thicker films. They attributed this observation to vacancy diffusion and annihilation at the free surface. We assumed that there are incohesive regions parallel to the in-plane direction and estimate their effect by replacing them with penny shaped microcracks. The elastic constants of such a two-phased composite $\left(C_{c}\right)$ are calculated using Eshelby's equivalent inclusion theory $^{13)}$ and Mori-Tanaka's mean field theory ${ }^{14)}$ as

$$
\begin{aligned}
& \mathbf{C}_{\mathbf{c}}=\mathbf{C}_{\mathbf{M}}+\left[f_{I}\left(\mathbf{C}_{\mathbf{I}}-\mathbf{C}_{\mathbf{M}}\right) \mathbf{A}_{\mathbf{d}}\right]\left[f_{M} \mathbf{I}+f_{I} \mathbf{A}_{\mathbf{d}}\right]^{-1}, \\
& \mathbf{A}_{\mathbf{d}}=\left[\mathbf{S C}_{\mathbf{M}}^{-1}\left(\mathbf{C}_{\mathbf{I}}-\mathbf{C}_{\mathbf{M}}\right)+\mathbf{I}\right]^{-1}
\end{aligned}
$$

where $\mathbf{C}_{\mathbf{M}}$ and $\mathbf{C}_{\mathbf{I}}$ are elastic-constant tensors of the matrix and inclusions, respectively, and $\mathbf{S}$ is Eshelby's tensor. $f_{M}$, and $f_{I}$ are volume fractions of the matrix and inclusion. Eshelby's tensor depends on the shape of the inclusion and Poisson's ratio of the isotropic matrix. We assumed zero modulus and $a_{1}: a_{2}: a_{3}=1: 1: 0.001$ for the inclusions $\left(a_{i}\right.$ are axes of the oblate-ellipsoid inclusions). When the porosity is $6.2 ! \_10^{-4}$, the calculation gives the diagonal components of the elastic constants close to those of the measurements as shown in Table I. Thus, only the incohesive regions can explain the occurrence of the $C_{33}$ smaller than $C_{11}$ and smaller $C_{i j}$ than those of the bulk material.

\section{Conclusion}

In this study, we established the RUS/laser measurement for the thin film $C_{i j}$ and determined the anisotropic elastic constants of $2.3 \mu \mathrm{m}$ copper film. The elastic constants of the copper film show anisotropy between the in-plane and out-of-plane directions. We conclude that the incohesive bonds caused this anisotropy.

1) A. Rouzaud, E. Barbier, J. Ernoult and E. Quesnel: Thin Solid Films 270 (1995) 270.

2) S. Sakai, H. Tanimoto and H. Mizubayashi: Acta Mater. 47 (1999) 211.

3) P. Djemia, F. Ganot, P. Moch, V. Branger and P. Goudeau: J. Appl. Phys. 90 (2001) 756.

4) I. Ohno: J. Phys. Earth 24 (1976) 355.

5) A. Migliori, J.L. Sarrao, W.M. Visscher, T.M. Bell, M. Lei, A. Fisk and R.G. Leisure: Physica B 183 (1993) 1.

6) H. Ogi, Y. Kawasaki, M. Hirao and H. Ledbetter: J. Appl. Phys. 92 (2002) 2451.

7) N. Nakamura, H. Ogi, T. Ichitsubo, M. Hirao, N. Tatsumi, T. Imai and H. Nakahata: J. Appl. Phys. 94 (2003) 6405.

8) N. Nakamura, H. Ogi and M. Hirao: Acta Mater. 52 (2004) 765.

9) P. Heyliger: J. Acoust. Soc. Am. 107 (2000) 1235.

10) H. Ogi, P. Heyliger, H. Ledbetter and S. Kim: J. Acoust. Soc. Am. 108 (2000) 2829.

11) G. Simmons and H. Wang: Single Crystal Elastic Constants and Calculated Aggregate Properties : a Handbook (The M.I.T. PRESS, Cambridge, 1971).

12) R. Schwaiger, G. Dehm and O. Kraft: Philos. Mag. 83 (2003) 693.

13) J.D. Eshelby: Proc. Roy. Soc. London, A241 (1957) 376.

14) T. Mori and K. Tanaka: Acta Metall. 21 (1973) 571. 